\title{
The CBOE S\&P 500 Three-Month Variance Futures
}

\author{
Jin E. Zhang ${ }^{1}$ \\ Associate Professor \\ School of Economics and Finance \\ The University of Hong Kong \\ Pokfulam Road, Hong Kong \\ People's Republic of China \\ Yuqin Huang \\ Doctoral student \\ School of Economics and Finance \\ The University of Hong Kong \\ Pokfulam Road, Hong Kong \\ People's Republic of China
}

First Version: January 2008

Final Version: January 2009

Forthcoming in Journal of Futures Markets

Keywords: Volatility trading; Variance Futures; VIX

JEL Classification Code: G13

This paper was previously circulated under the title "The Market for Volatility Trading: Variance Futures". The author acknowledge helpful comments from an anonymous referee, Bob Webb (editor), and seminar participants at the University of Hong Kong and the 2008 Financial Management Association (FMA) Annual Meeting. Jin E. Zhang's work has been supported by a grant from the Research Grants Council of the Hong Kong Special Administrative Region, China (Project No. HKU 7427/06H).

\footnotetext{
${ }^{1}$ Corresponding author, School of Economics and Finance, the University of Hong Kong, Pokfulam Road, Hong Kong, People's Republic of China; Tel: (852) 2859 1033, Fax: (852) 2548 1152, Email: jinzhang@hku.hk
} 


\title{
The CBOE S\&P 500 Three-Month Variance Futures
}

\author{
Abstract \\ In this paper, we study the market of the CBOE S\&P 500 three-month variance futures \\ that were listed on May 18, 2004. By using a simple mean-reverting stochastic volatility \\ model for the S\&P 500 index, we present a linear relation between the price of fixed \\ time-to-maturity variance futures and the $V I X^{2}$. The model prediction is supported by \\ empirical tests. We find that a model with a fixed mean-reverting speed of 1.2929 and a \\ daily-calibrated floating long-term mean level has a good fit to the market data between \\ May 18, 2004 and August 17, 2007. The market price of volatility risk estimated from the \\ 30-day realized variance and $V I X^{2}$ has a mean value of -19.1184 .
}




\section{Introduction}

Volatility/variance has become an asset class in its own right. In late 1990s, Wall Street firms started trading variance swaps, forward contracts written on the realized variance. These swaps are now the preferred route for many hedge fund managers and proprietary traders to make bets on market volatility. According to some estimates, the daily trading volume in equity-index variance swaps reached USD 4-5 million vega notional in 2006. On an annual basis, this corresponds to payments of more than USD 1 billion per percentage point of volatility (Carr and Lee 2007). The trading volume of the over-the-counter (OTC) variance swaps market seems small relative to many markets, but the volatility derivatives market has great potential due to the large demand for trading and hedging volatility risk (Brenner, Ou and Zhang 2006).

The exchange-listed volatility products started with the Chicago Board Options Exchange (CBOE) volatility index (VIX) futures in March 2004. Currently, there are six kinds of volatility futures and three kinds of volatility options traded on the CBOE. There are three kinds of volatility futures traded on the Eurex. They are derivative contracts written on either the volatility index or realized variance. Table 1 provides a brief summary of these products.

The study on the exchange market for volatility trading has been growing rapidly in the last few years. Zhang and Zhu (2006) first model the VIX and VIX futures by using Heston (1993) model for the instantaneous variance and test their VIX futures pricing model by using market data on March 1, 2005. Zhu and Zhang (2007) extend Zhang and Zhu (2006) by allowing long-term mean level to be time-dependent, test their new VIX futures pricing model by using market data on March 10, 2005. Lin (2007) use affine jump-diffusion model with jumps in index and volatility, and study VIX futures prices between April 21, 2004 to April 18, 2006. Brenner, Shu and Zhang (2006) derive a new approximate analytical 
VIX futures price formula under Zhang and Zhu's (2006) setup, study VIX futures market prices between March 26, 2004 and November 21, 2006. Sepp (2008a, b) use affine jumpdiffusion model with jump in volatility, study VIX futures and VIX option pricing model, and estimate model by using daily VIX levels from February 28, 2003 to February 29, 2008. These studies mainly focus on the VIX futures market ${ }^{2}$. However, the variance futures market has never been discussed in the literature. It is the focus of this paper.

Variance futures are futures contracts written on realized variance. They can be regarded as standardized variance swaps. There are two types of variance futures, threemonth and 12-month futures, that are currently traded on the CBOE. They were first listed on the CBOE Futures Exchange (CFE) on May 18, 2004 and March 23, 2006 respectively.

Currently the VIX futures and options are the most liquid exchange-listed volatility products. The S\&P 500 (SPX) three-month variance futures are much less liquid than the VIX futures. For example, on November 21, 2007, the trading volume of the VIX futures was 3,303 contracts, which was 40 times greater than 76 contracts, which was the trading volume of the SPX three-month variance futures. For the period between May 18, 2004 and August 17, 2007, the average open interest of SPX three-month variance futures was 217 contracts, while the average daily trading volume was 4.7 contracts. We show here that a variance futures contract is much simpler in terms of pricing, because it can be replicated by a portfolio of the S\&P 500 index options. It is puzzling to us why the trading volume

\footnotetext{
${ }^{2}$ Other related literatures are as follows. Carr and Wu (2006) tell the story how the CBOE developed its new volatility index, VIX. They propose a theory to calculate an upper bound for VIX futures price, and the variance of the VIX at future time by using current prices of European options with different strikes. Dotsis, Psychoyios and Skiadopoulos (2007) study the jump diffusion models for the volatility indices, such as VIX, VXO, VXD, VDAX and VX1/VX6. Jiang and Tian (2007) examine the robustness of the CBOE procedure in calculating the new VIX. Konstantinidi, Skiadopoulos and Tzagkaraki (2008) address the question whether the evolution of implied volatility can be forecasted by studying a number of European and US implied volatility indices. Carr and Wu (2009) study the variance risk premiums of individual stock options market. Duan and Yeh (2007) develop an estimation method for extracting the latent stochastic volatility from VIX.
} 
of SPX three-month variance futures is much lower than that of VIX futures, which seem to be a more complicated product.

The difference between three-month and 12-month variance futures is the length of the period to calculate the variance. We use three-month futures as an example to describe the nature of the contract. The final settlement value of a three-month variance futures contract is the annualized realized variance of the $\mathrm{S} \& \mathrm{P} 500$ index within the three months before the maturity multiplied by 10,000. The formula to calculate the annualized realized variance $(R V)$ is given by

$$
R V=252 \times \sum_{i=1}^{N_{a}-1} \frac{R_{i}^{2}}{N_{e}-1} .
$$

In this formula, $R_{i}=\ln \left(S_{i+1} / S_{i}\right)$ is the daily continuously compounded return of the $\mathrm{S} \& \mathrm{P}$ 500 index. $S_{i+1}$ is the final value of the $\mathrm{S} \& \mathrm{P} 500$ index to calculate the daily return. $S_{i}$ is the initial value of the S\&P 500 index to calculate the daily return. $N_{e}$ is the number of expected S\&P 500 values needed to calculate daily returns during the three-month period. The total number of daily returns expected during the three-month period is $N_{e}-1 . N_{a}$ is the actual number of $\mathrm{S} \& \mathrm{P} 500$ values used to calculate daily returns during the threemonth period. Generally, the actual number of S\&P 500 values will equal the expected number of S\&P 500 values (represented by $N_{e}$ ). However, if one or more "market disruption events" occurs during the three-month period, the actual number of S\&P 500 values will be less than the expected number of S\&P 500 values by an amount equal to the number of market disruption events that occurred during the three-month period. The total number of actual daily returns during the three-month period is $N_{a}-1$. During the calculation of the realized variance, the average daily return is assumed to be zero. The daily return squared is used as a proxy for the daily variance. This practice has become an industry standard in calculating the settlement value of variance swaps.

The three-month realized variance computed from formula (1) is not a traded asset, 
even though it is observable. Therefore, the variance futures cannot be priced relatively by the no-arbitrage principle. The variance futures price is the expected value of future realized variance in a risk-neutral measure. The variance futures price process will provide us useful information on the risk-neutral dynamics of instantaneous variance.

In Section 2, we present a simple model for the variance futures price based on the Heston (1993) stochastic variance model for the S\&P 500 index. The model links the three-month variance futures price with the VIX. The data is described in Section 3. The model is tested in Section 4. The paper is concluded in Section 5.

\section{The model}

We now use a simple model to establish the relationship between variance futures prices and instantaneous variance. Since the VIX is also related to instantaneous variance, we can build a relation between variance futures prices and VIX.

In the physical measure, $\mathcal{P}$, the $\mathrm{S} \& \mathrm{P} 500$ index (SPX), $S_{t}$, is assumed to follow the Heston (1993) stochastic volatility model as follows:

$$
\begin{aligned}
\frac{d S_{t}}{S_{t}} & =\mu d t+\sqrt{V_{t}} d B_{1 t}^{P}, \\
d V_{t} & =\kappa^{P}\left(\theta^{P}-V_{t}\right) d t+\sigma_{V} \sqrt{V_{t}} d B_{2 t}^{P},
\end{aligned}
$$

where $\mu$ is the expected return on an investment in the SPX index and $V_{t}$ is the instantaneous variance. In general, the instantaneous variance is not observable. It has to be recovered from some other observable volatility process, such as VIX process. The parameter $\kappa^{P}$ is the mean-reverting speed; $\theta^{P}$ is the long-term mean level; and $\sigma_{V}$ measures the volatility of the variance process. The increments of the two standard $\mathcal{P}$ Brownian motions, $d B_{1 t}^{P}$ and $d B_{2 t}^{P}$, model the uncertainties within the time period from $t$ to $t+d t$ in the SPX index and the instantaneous variance. They are assumed to be correlated with a constant coefficient, $\rho$. 
We change the probability measure from the physical one, $\mathcal{P}$, to the risk-neutral one, $\mathcal{Q}$, as follows:

$$
d B_{1 t}^{P}=d B_{1 t}^{Q}-\frac{\mu-r}{\sqrt{V_{t}}} d t, \quad d B_{2 t}^{P}=d B_{2 t}^{Q}-\frac{\lambda}{\sigma_{V}} \sqrt{V_{t}} d t
$$

where $r$ is the risk-free rate and $\lambda$ is the market price of volatility risk. We obtain the dynamics of the SPX index in the risk-neutral measure

$$
\begin{aligned}
& \frac{d S_{t}}{S_{t}}=r d t+\sqrt{V_{t}} d B_{1 t}^{Q}, \\
& d V_{t}=\kappa\left(\theta-V_{t}\right) d t+\sigma_{V} \sqrt{V_{t}} d B_{2 t}^{Q},
\end{aligned}
$$

where $d B_{1 t}^{Q}$ and $d B_{2 t}^{Q}$ are the increments of two standard $\mathcal{Q}$ Brownian motions with the correlation coefficient, $\rho$. The risk-neutral mean-reverting speed, $\kappa$, and long-term mean level, $\theta$, are related to the physical parameters as follows

$$
\kappa=\kappa^{P}+\lambda, \quad \theta=\frac{\kappa^{P} \theta^{P}}{\kappa^{P}+\lambda} .
$$

From equation (6), we immediately have the conditional mean of the future instantaneous variance:

$$
E_{t}^{Q}\left(V_{s}\right)=\theta\left(1-e^{-\kappa(s-t)}\right)+V_{t} e^{-\kappa(s-t)},
$$

which is a weighted average between $\theta$ and $V_{t}$, with $e^{-\kappa(s-t)}$ as the weight. It can be regarded as the price of futures written on the instantaneous variance.

\subsection{VIX and the variance risk premium}

The CBOE volatility index, VIX, is defined as the 30 -day variance swap rate ${ }^{3}$. The relationship between VIX squared $\left(V I X^{2}\right)$ and the instantaneous variance has been established

\footnotetext{
${ }^{3}$ The details of computing the VIX from the SPX options prices are available in the CBOE white paper, available at: http://www.cboe.com/micro/vix/vixwhite.pdf. According to market practice, the variance swap rate is quoted as the volatility (the square root of the variance) instead of the variance.
} 
by Zhang and Zhu (2006) as follows

$$
\begin{aligned}
\left(\frac{V I X_{t}}{100}\right)^{2} & =E_{t}^{Q}\left(\frac{1}{\tau_{0}} \int_{t}^{t+\tau_{0}} V_{s} d s\right)=\frac{1}{\tau_{0}} \int_{t}^{t+\tau_{0}} E_{t}^{Q}\left(V_{s}\right) d s \\
& =\frac{1}{\tau_{0}} \int_{t}^{t+\tau_{0}}\left[\theta+\left(V_{t}-\theta\right) e^{-\kappa(s-t)}\right] d s \\
& =\left(1-B_{0}\right) \theta+B_{0} V_{t}, \quad \text { where } \quad B_{0}=\frac{1-e^{-\kappa \tau_{0}}}{\kappa \tau_{0}}, \quad \tau_{0} \equiv \frac{30}{365}
\end{aligned}
$$

For a positive $\kappa, B_{0}$ is a number between 0 and 1 . The $V I X^{2}$ is the weighted average between the risk-neutral long-term mean level, $\theta$, and the instantaneous variance, $V_{t}$, with $B_{0}$ as the weight. VIX $X^{2}$ can be regarded as the price of an always-30-day variance futures price.

The variance risk premium (VRP), measured by the return of a 30-day variance swap (Carr and Wu 2009), is given by

$$
\begin{aligned}
V R P & =E_{t}^{P}\left(\frac{1}{\tau_{0}} \int_{t}^{t+\tau_{0}} V_{s} d s\right)-E_{t}^{Q}\left(\frac{1}{\tau_{0}} \int_{t}^{t+\tau_{0}} V_{s} d s\right) \\
& =\left(1-B_{0}^{P}\right) \theta^{P}+B_{0}^{P} V_{t}-\left(1-B_{0}\right) \theta-B_{0} V_{t},
\end{aligned}
$$

where $B_{0}^{P}=\frac{1-e^{-\kappa^{P} \tau_{0}}}{\kappa^{P} \tau_{0}}$. Substituting $\kappa^{P} \theta^{P}=\kappa \theta$ and $\kappa^{P}+\lambda=\kappa$ into equation (10) gives

$$
\begin{aligned}
V R P= & {\left[\frac{\kappa}{\kappa^{P}}\left(1-B_{0}^{P}\right)-\left(1-B_{0}\right)\right] \theta+\left(B_{0}^{P}-B_{0}\right) V_{t} } \\
= & {\left[\frac{\kappa}{\kappa-\lambda}\left(1-\frac{1-e^{-(\kappa-\lambda) \tau_{0}}}{(\kappa-\lambda) \tau_{0}}\right)-\left(1-\frac{1-e^{-\kappa \tau_{0}}}{\kappa \tau_{0}}\right)\right] \theta } \\
& \quad+\left[\frac{1-e^{-(\kappa-\lambda) \tau_{0}}}{(\kappa-\lambda) \tau_{0}}-\frac{1-e^{-\kappa \tau_{0}}}{\kappa \tau_{0}}\right] V_{t} \\
= & {\left[\frac{\kappa \tau_{0}\left(1+e^{-\kappa \tau_{0}}\right)-2\left(1-e^{-\kappa \tau_{0}}\right)}{\kappa^{2} \tau_{0}^{2}} \theta+\frac{1-e^{-\kappa \tau_{0}}-\kappa \tau_{0} e^{-\kappa \tau_{0}}}{\kappa^{2} \tau_{0}^{2}} V_{t}\right] \lambda \tau_{0}+O\left(\lambda^{2} \tau_{0}^{2}\right) } \\
= & {\left[\left(\frac{1}{6} \kappa \tau_{0}+O\left(\kappa^{2} \tau_{0}^{2}\right)\right) \theta+\left(\frac{1}{2}-\frac{1}{3} \kappa \tau_{0}+O\left(\kappa^{2} \tau_{0}^{2}\right)\right) V_{t}\right] \lambda \tau_{0}+O\left(\lambda^{2} \tau_{0}^{2}\right), }
\end{aligned}
$$

where the last two equalities are due to the Taylor expansion for small $\lambda \tau_{0}$ and $\kappa \tau_{0}$. The variance risk premium is almost proportional to the market price of volatility risk, $\lambda$, if $\lambda \tau_{0}$ is small. 


\section{$2.2 \quad$ Variance futures}

We consider three-month variance futures with maturity at $T$. The contract will be settled at $T$ with the realized variance between $T-\tau_{1}$ and $T$, where $\tau_{1}=3$ months $=1 / 4$ year.

For forward-starting variance futures, $0<t<T-\tau_{1}$, the futures price is given by

$$
\begin{aligned}
\frac{F_{t}^{T}}{10,000} & =E_{t}^{Q}\left(\frac{1}{\tau_{1}} \int_{T-\tau_{1}}^{T} V_{s} d s\right)=\frac{1}{\tau_{1}} \int_{T-\tau_{1}}^{T} E_{t}^{Q}\left(V_{s}\right) d s \\
& =\frac{1}{\tau_{1}} \int_{T-\tau_{1}}^{T}\left[\theta+\left(V_{t}-\theta\right) e^{-\kappa(s-t)}\right] d s \\
& =(1-B) \theta+B V_{t}, \quad \text { where } \quad B=\frac{1-e^{-\kappa \tau_{1}}}{\kappa \tau_{1}} e^{-\kappa\left(T-\tau_{1}-t\right)}
\end{aligned}
$$

For a positive $\kappa, B$ is a number between 0 and 1 , and $B \rightarrow 0$ if $T \rightarrow+\infty$. The price of very long maturity variance futures is equal to the risk-neutral long-term mean level of the variance. Combining equations (9) and (12) gives a relationship between the price of forward-starting variance futures and the VIX index:

$$
\frac{F_{t}^{T}}{10,000}=\left(1-\frac{B}{B_{0}}\right) \theta+\frac{B}{B_{0}}\left(\frac{V I X_{t}}{100}\right)^{2},
$$

which is a weighted average between the long-term mean level, $\theta$, and $\left(\frac{V I X_{t}}{100}\right)^{2}$ with $\frac{B}{B_{0}}$ as the weight.

For backward-starting variance futures, $T-\tau_{1}<t<T$, the annualized realized variance between $T-\tau_{1}$ and $t$ is given by

$$
R V=252 \times \sum_{i=1}^{N} \frac{R_{i}^{2}}{N},
$$

where $N+1$ is the total number of days between $T-\tau_{1}$ and $t$. The futures price is then 
given by

$$
\begin{aligned}
\frac{F_{t}^{T}}{10,000} & =\frac{1}{\tau_{1}}\left[\left(\tau_{1}-T+t\right) R V+E_{t}^{Q}\left(\int_{t}^{T} V_{s} d s\right)\right]=\left(1-\frac{T-t}{\tau_{1}}\right) R V+\frac{1}{\tau_{1}} \int_{t}^{T} E_{t}^{Q}\left(V_{s}\right) d s \\
& =\left(1-\frac{T-t}{\tau_{1}}\right) R V+\frac{1}{\tau_{1}} \int_{t}^{T}\left[\theta+\left(V_{t}-\theta\right) e^{-\kappa(s-t)}\right] d s \\
& =\left(1-\frac{T-t}{\tau_{1}}\right) R V+\frac{T-t}{\tau_{1}}\left[\left(1-B^{*}\right) \theta+B^{*} V_{t}\right], \\
& =\left(1-\frac{T-t}{\tau_{1}}\right) R V+\frac{T-t}{\tau_{1}}\left[\left(1-\frac{B^{*}}{B_{0}}\right) \theta+\frac{B^{*}}{B_{0}}\left(\frac{V I X_{t}}{100}\right)^{2}\right],
\end{aligned}
$$

where $B^{*}=\frac{1-e^{-\kappa(T-t)}}{\kappa(T-t)}$. It is a weighted average between the realized variance $(R V)$, the long-term mean level, $\theta$, and $\left(\frac{V I X_{t}}{100}\right)^{2}$.

The observable VIX can be regarded as the underlying financial variable of the variance futures.

\section{Data}

We construct 30-day and three-month historical variance (HV) by using formula (1) with the daily closing level of the S\&P 500 index. The daily S\&P 500 three-month variance futures data provided by the CBOE are available from May 18, 2004 to the present.

For each day, we have four variance futures contracts on the March quarterly cycle. For example, on the first day of the listing, May 18, 2004, four contracts, U4, Z4, H5 and M5, were traded. These names indicate the following futures expirations: September and December 2004, and March and June 2005 respectively. The first letter indicates the expiration month ${ }^{4}$ followed by the expiration year. The variance futures contract is quoted in terms of variance points. Variance points are defined as the realized variance multiplied by 10,000 . The contract size is $\$ 50$ times the variance points. For example, a variance

\footnotetext{
${ }^{4}$ The expiration month codes follow the convention for all commodities futures, which is defined as follows: January-F, February-G, March-H, April-J, May-K, June-M, July-N, August-Q, September-U, October-V, November-X and December-Z.
} 
calculation of 0.06335 would have a corresponding price quotation in variance points of 633.50 , and the contract size would be $\$ 31,675.00(633.50 \times \$ 50)$. The final settlement date is the third Friday of the expiration month.

Our empirical study covers the period of three years and three months from May 18, 2004 to August 17, 2007, within which there were 16 contract months traded. Table 2 provides the summary statistics of all contracts. For all contract months, the average open interest was 217 contracts, while the average daily trading volume was 4.7 contracts. Normally the contracts lasted for a year. The average variance futures price changed from 228.6 for contracts that matured in September 2004 to 361.4 for contracts that matured in June 2008 (the average was made on samples up to the maturity date or August 17, 2007, whichever was earlier), while the VIX index level changed from 19.33 on May 18, 2004 to 29.99 on August 17, 2007. The expected future volatility increased dramatically in July and August 2007 when the subprime mortgage crisis came to light.

\section{Empirical evidence}

\subsection{The relationship between variance futures and VIX}

As described in equation (13), with the assumption that the instantaneous variance is mean-reverting, we can establish a linear relationship between a forward-starting threemonth variance futures price and the VIX as follows

$$
F_{t}^{T}=\alpha(\tau)+\beta(\tau) V I X_{t}^{2}, \quad \tau \equiv T-t \geq \tau_{1} \equiv 1 / 4 \text { year }
$$

where

$$
\alpha(\tau)=10,000 \times[1-\beta(\tau)] \theta, \quad \beta(\tau)=\frac{\tau_{0}}{\tau_{1}} \frac{1-e^{-\kappa \tau_{1}}}{1-e^{-\kappa \tau_{0}}} e^{-\kappa\left(\tau-\tau_{1}\right)}, \quad \tau_{0} \equiv \frac{30}{365} .
$$

To test this relation, we need to construct a fixed time-to-maturity variance futures series. 
There are four variance futures contracts available on a typical day. For example, on May 18, 2004, there were variance futures contracts with maturities in September and December 2004 and March and June 2005, which correspond to times to maturity of 85, 149, 211 and 274 trading days. We construct three-month, six-month and nine-monthto-maturity variance futures prices by a linear interpolation technique. For example, on May 18, 2004, the three-month-to-maturity (63 trading days) variance futures price is computed by using the market data of three-month historical variance ${ }^{5}$ and September futures. The six-month-to-maturity (126 trading days) variance futures price is computed with September and December futures. The nine-month-to-maturity (189 trading days) variance futures price is computed with December and March futures. We calculate the fixed time-to-maturity three-month variance futures prices on each day and obtain three time series. Table 3 provides the summary statistics of their levels and returns. The returns are computed as the logarithm of the price relative on two consecutive end-of-day prices. Both the levels and returns of the fixed time-to-maturity variance futures are positively skewed. Figure 1 shows the time series of three-month historical variance HV (the threemonth historical variance at time $t, H V_{t}$, stands for the annual variance calculated with equation (1) for the period between $t-3 / 12$ and $t$ ), VIX ${ }^{2}$ and variance futures with three fixed times-to-maturity. Table 4 presents the correlation matrix between the levels and returns of the S\&P 500 index, VIX, VIX ${ }^{2}, 30$-day and three-month historical variance and variance futures. The three futures series are negatively correlated with the S\&P 500 index. The variance futures with three different times-to-maturity are very highly correlated. They are also positively correlated with VIX and VIX ${ }^{2}$. Figure 1 also shows that the trading volume of the variance futures has been slowly increasing.

We now explore the relationship between variance futures and VIX ${ }^{2}$ or historical variance using the market data from May 18, 2004 to August 17, 2007. We examine the

\footnotetext{
${ }^{5}$ The three-month historical variance can be regarded as variance futures with 0 day to maturity.
} 
relationship using the following equation:

$$
F_{t}^{T}=\alpha+\beta V_{t}+\varepsilon_{t}
$$

with fixed time-to-maturity, $\tau \equiv T-t$, where $V_{t}$ could be VIX ${ }^{2}$ or three-month (30-day) historical variance. The regression results are reported in Table 5. The results in the table indicate that all linear regressions are significant at the one percent critical level. The coefficients of VIX ${ }^{2}$, three-month HV, 30-day HV are positive and strongly significant. From the $R^{2}$ value, we find that VIX ${ }^{2}$ has better explanatory power than three-month HV and 30-day HV. And futures prices with shorter maturities have stronger relations with the variables.

\subsection{Variance mean-reversion parameters}

For three-month-to-maturity variance futures, $\tau=1 / 4$, we obtain from regression that

$$
F_{t}^{t+1 / 4}=81.34+0.5993 V I X_{t}^{2}
$$

With $\alpha(\tau)=81.34$ and $\beta(\tau)=0.5993$, solving equation (17) for $\kappa$ and $\theta$, we find the values of the mean-reversion parameters of the variance,

$$
\kappa=7.684, \quad \theta=0.020300 .
$$

Similarly, with the regression result of six-month-to-maturity variance futures,

$$
F_{t}^{t+1 / 2}=143.86+0.5450 V I X_{t}^{2}
$$

we obtain another set of parameters,

$$
\kappa=1.8413, \quad \theta=0.031618
$$

With the regression result of nine-month-to-maturity variance futures,

$$
F_{t}^{t+3 / 4}=168.12+0.5241 V I X_{t}^{2},
$$


we obtain the third set of parameters,

$$
\kappa=1.1114, \quad \theta=0.035327
$$

The last two sets of parameters are very close, whereas the first set is not close to the last two.

Figure 1 and Table 3 show that the three-month-to-maturity variance futures prices are relatively low compared with the six- and nine-month-to-maturity variance futures prices. The average prices of the three fixed time-to-maturity variance futures contracts are 196.06, 248.18 and 268.44. The difference between the three-month and six-month prices is 52.12, which is much higher than 20.26, the difference between the six-month and nine-month prices. This is because when we construct the three-month-to-maturity variance futures price, we sometimes use information on realized variance. The realized variance is smaller than the implied variance due to the negative variance risk premium. Our construction procedure might underestimate the values of three-month-to-maturity variance futures prices.

We now study the stability of the parameters, $(\kappa, \theta)$. On each day, we calibrate the variance futures pricing model by using the market prices of four variance futures with different maturity dates and obtain a time series of the parameters. As observed in the top two graphs of Figure 3, the values of these parameters are not constant. They sometimes change dramatically. This calibration exercise shows that our simple model based on constant parameters might not be good enough to capture the dynamics of variance.

With the daily calibrated set of parameters, we can recalculate the prices of three fixed time-to-maturity variance futures contracts by using our pricing model (16). The results are shown graphically in the left column of Figure 4 together with those obtained with linear interpolation technique. The mean values of the three variance futures contracts calculated with our calibrated model are 210.29, 249.25 and 268.33, while the mean values determined 
by the linear interpolation technique are 196.06, 248.18 and 268.44. The difference between the prices of three-month-to-maturity variance futures contracts calculated by the two methods is quite large (around 7\%). The difference between the other two variance futures contracts is negligibly small. This exercise shows that the linear interpolation technique indeed underestimates three-month-to-maturity variance futures prices.

With the model-fitted three-month-to-maturity variance futures price, $F_{t}^{t+1 / 4}$, we run the linear regression against $V I X_{t}^{2}$ again and obtain the following parameters:

$$
\kappa=3.4781, \quad \theta=0.027234
$$

which are closer to the parameters obtained from six- and nine-month variance futures.

Finally, we solve following minimization problem:

$$
\min _{(\kappa, \theta)} \sum_{i=1}^{I} \sum_{j=1}^{4}\left(F_{t_{i} \text { model }}^{T_{j}}(\kappa, \theta)-F_{t_{i} \text { market }}^{T_{j}}\right)^{2},
$$

where index $i$ stands for $i$ th day, index $j$ stands for $j$ th contract on a particular day, $I=811$ is the total number of days in the sample. We obtain an optimal set of parameters,

$$
\kappa=1.2929, \quad \theta=0.034151
$$

calibrated unconditionally.

In the top two graphs of Figure 3, we observe that the dramatic changes in $\kappa$ and $\theta$ are often coupled with each other, which suggests that there might exist multiple solutions in the calibration exercise, i.e., minimizing the sum of the squared errors of four variance futures contracts. To test this conjecture, we fix $\kappa=1.2929$ and calibrate $\theta$. The result in the lower graph of Figure 3 is much more stable than before. We further calculate the prices of three-, six- and nine-month variance futures with the fixed $\kappa$ and floating $\theta$ and present them together with the results from the floating $\kappa$ and $\theta$ in Figure 4 . The results show that the method of keeping $\kappa$ fixed provides a stable $\theta$ and reasonably accurate variance futures 
prices. Our empirical study suggests that the following model for the S\&P 500 index:

$$
\begin{aligned}
\frac{d S_{t}}{S_{t}} & =r d t+\sqrt{V_{t}} d B_{1 t}^{Q} \\
d V_{t} & =\kappa\left(\theta_{t}-V_{t}\right) d t+\sigma_{V} \sqrt{V_{t}} d B_{2 t}^{Q} \\
d \theta_{t} & =\sigma_{\theta} d B_{3 t}^{Q}
\end{aligned}
$$

is probably the simplest but a reasonably good choice for the variance futures pricing.

\subsection{Variance risk premium}

The 30-day historical variance at time $t, H V_{t}$, stands for the annual variance calculated with equation (1) for the period between $t-30 / 365$ and $t$. The subsequent 30-day realized variance at time $t, R V_{t}$, stands for the annual variance calculated with equation (1) for the period between $t$ and $t+30 / 365$. The variance risk premium, discussed in Section 2.1, is the difference between $R V_{t}$ and $V I X_{t}^{2}$, which is the dollar return on 30-day variance swaps.

The time series of 30-day historical variance, realized variance and $V I X^{2}$ are shown in the top graph of Figure 5. The variance risk premium, shown in the lower graph of Figure 5 , is negative most of the time. It becomes positive in the recent months of February and July 2007. The average value of the variance risk premium is -66.0413 .

With the fixed $\kappa$ calibrated from the whole sample, $\kappa=1.2929$, and a daily calibrated floating $\theta$, we can calculate the instantaneous variance, $V_{t}$, from the time series of $V I X_{t}$ with equation (9), which is shown in the top graph of Figure 6. We observe that the processes of $V_{t}$ and $V I X_{t}^{2}$ are highly correlated with each other. Finally, we calculate the market price of volatility risk, $\lambda$, with equation (11). It is negative most of the time as shown in the lower graph in Figure 6 with an average value of -19.1184.

The values of the parameters $\kappa, \theta_{t}, V_{t}$ and $\lambda_{t}$ calibrated from the variance futures market should be used to price S\&P 500 index options and other volatility derivatives, such as VIX futures and options. 


\section{Conclusions}

There are two types of volatility/variance derivatives that have been traded on exchanges. One is written on the realized variance. The other one is written on some kind of implied volatility, such as the Chicago Board Options Exchange (CBOE) volatility index, VIX.

In this paper, we study the market of three-month variance futures by using the CBOE price data from May 17, 2004 (the listing date) to August 17, 2007. We use the Heston (1993) stochastic variance model for the SPX index and present a formula for the forwardand backward-starting variance futures prices. By using Zhang and Zhu's (2006) formula for the VIX, we link the variance futures prices with the observable variable, VIX. The main prediction of the model is that the fixed time-to-maturity variance futures price is a linear function of the $V I X^{2}$.

We construct three-, six- and nine-month-to-maturity variance futures by using the available market data with a linear interpolation technique. Our regression results show that the linear relation between the variance futures price and $V I X^{2}$ is significant at the one percent critical level. Our calibration exercises show that a model with fixed meanreverting speed, $\kappa$ (1.2929 calibrated unconditionally), and a floating long-term mean level, $\theta$ (calibrated daily), has a good (as good as a floating $\kappa$ and $\theta$ ) fit with the market data. We also estimate the volatility risk premium and the market price of volatility risk from the difference between the realized variance and $V I X^{2}$. Our estimation shows that the volatility risk premium is negative most of the time. The average value of the market price of volatility risk is -19.1184 .

In this paper, the parameters for the simple mean-reverting process are derived directly from the variance futures market. They are important information for us to understand the sophisticated dynamics of market volatility. They should be used in studying the consistency between the S\&P 500 index options market and the VIX futures and options 
market.

Here, we would like to make three comments. The first comment is on estimating the parameters in the volatility model. Our parameters $(\kappa, \lambda)=(1.2929,-19.1184)$ are estimated from the variance futures market, SPX realized variance and VIX for the period between May 18, 2004 and August 17, 2007. They are different from those in Lin (2007), $(5.3500,-0.3528)^{6}$, for the period between April 21, 2004 and April 18, 2006, and those in Duan and Yeh $(2007),(-1.7986,-7.5697)^{7}$, for the period between January 2, 2001 and December 29, 2006. Our volatility risk premium, more than twice as large as Duan and Yeh's, is 50 times larger than Lin's. Our volatility process is mean-reverting in riskneutral measure, but Duan and Yeh's is not. The questions why the parameters in the same Heston (1993) model estimated with different approaches are so different and what the most reliable way is to estimate the parameters in the volatility process remain open.

The second comment is on the mathematical tools in modeling the volatility process. Our formulas for the VIX, variance risk premium and variance futures price are derived under Heston (1993) model, i.e., the risk-neutral instantaneous variance in equation (6) is modeled with mean-reverting squared-root process. But our results still hold if the increment of the Brownian motion, $d B_{2 t}^{Q}$, is replaced by some other kinds of Lévy process (Carr and Wu 2004) with zero mean, including the jump-diffusion. In other word, the variance and skewness of the incremental random noise in the risk-neutral volatility process does not have an influence on the variance futures market. The major factors that drive the volatility futures market are short-term variance, $V_{t}$, and long-term variance, $\theta_{t}$.

The third comment is on the correlation coefficient, $\rho$, between the SPX index and the instantaneous variance. It seems to us that this factor does not enter into the variance

\footnotetext{
${ }^{6}$ The risk-neutral mean-reverting speed, $\kappa$, is computed as $\kappa_{v}+\eta_{v}$, where $\kappa_{v}=5.7028$ and $\eta_{v}=-0.3528$ from Lin's (2007) Table II. The market price of volatility risk, $\lambda$, is the same as Lin's $\eta_{v}=-0.3528$.

${ }^{7}$ The parameter values are from $\kappa^{*}$ and $\delta_{V}$ of SV2 model in the last panel of Table 2 of Duan and Yeh (2007).
} 
futures market even though it is crucial in determining the risk-neutral skewness of the index return observed in the SPX options market as the phenomenon of the implied volatility smirk (Zhang and Xiang 2008). 


\section{References}

[1] Brenner, M., Ou, E. Y., \& Zhang, J. E. (2006). Hedging volatility risk. Journal of Banking and Finance, 30, 811-821.

[2] Brenner, M., Shu, J.-H., \& Zhang, J. E. (2006). The market for volatility trading; VIX futures (working paper). New York University.

[3] Carr, P., \& Wu, L.-R. (2004). Time-changed Lévy processes and option pricing. Journal of Financial Economics, 17(1), 113-141.

[4] Carr, P., \& Wu, L.-R. (2006). A tale of two indices. Journal of Derivatives, 13, 13-29.

[5] Carr, P., \& Wu, L.-R. (2009). Variance risk premiums. Review of Financial Studies, (forthcoming).

[6] Carr, P., \& Lee, R. (2007). Robust replication of volatility derivatives (working paper). Bloomberg LP and University of Chicago.

[7] Dotsis, G., Psychoyios, D., \& Skiadopoulos, G. (2007). An empirical comparison of continuous-time models of implied volatility indices. Journal of Banking and Finance, 31, 3584-3603.

[8] Duan, J.-C., \& Yeh, C.-Y. (2007). Jump and volatility risk premium implied by VIX (working paper). University of Toronto.

[9] Heston, S. L. (1993). A closed-form solution for options with stochastic volatility with applications to bond and currency options. Review of Financial Studies, 6(2), 327-343.

[10] Huang, Y.-Q., \& Zhang, J. E. (2008). The market for volatility trading: Variance futures. Paper presented in 2008 Financial Management Association (FMA) Annual Meeting, October 8-11, 2008, Grapevine (Dallas), Texas, U.S.A. 
[11] Jiang, G. J., \& Tian, Y. S. (2007). Extracting model-free volatility from option prices: An examination of the VIX index. Journal of Derivatives, 14(3), 1-26.

[12] Konstantinidi, E., Skiadopoulos G., \& Tzagkaraki, E. (2008). Can the evolution of implied volatility be forecasted? Evidence from European and US implied volatility indices. Journal of Banking and Finance, 32, 2401-2411.

[13] Lin, Y.-N. (2007). Pricing VIX futures: Evidence from integrated physical and riskneutral probability measures. Journal of Futures Markets, 27(12), 1175-1217.

[14] Sepp, A. (2008a). VIX option pricing in jump-diffusion model. Risk, April 2008, 84-89.

[15] Sepp, A. (2008b). Pricing options on realized variance in Heston model with jumps in returns and volatility. Journal of Computational Finance, 11(4), 33-70.

[16] Zhang, J. E., \& Xiang, Y. (2008). The implied volatility smirk. Quantitative Finance, $8(3), 263-284$.

[17] Zhang, J. E., \& Zhu, Y.-Z. (2006). VIX futures. Journal of Futures Markets, 26(6), $521-531$.

[18] Zhu, Y.-Z., \& Zhang, J. E. (2007). Variance term structure and VIX futures pricing. International Journal of Theoretical and Applied Finance, 10(1), 111-127. 


\section{Table 1}

\section{Brief Summary of Exchange-listed Volatility/Variance Products}

There are twelve volatility/variance derivative products listed in the CBOE Futures Exchange (CFE) and the Eurex. In Panel A, we provide information on the CFE volatility futures; in Panel $\mathrm{B}$, we provide the information on the CBOE volatility options. In Panel $\mathrm{C}$, we provide information on the Eurex volatility futures. The information on trade volume and open interest is the number of contracts on a randomly-chosen date: November $21,2007$.

\begin{tabular}{lccc} 
Products & Trade Volume & Open Interest & Listing Date \\
\hline Panel A: The CFE volatility/variance futures & & & \\
Nasdaq-100 Volatility Index (VXN) & 5 & 55 & Jul. 6, 2007 \\
Russell 2000 Volatility Index (RVX) & 50 & 2,467 & Jul. 6, 2007 \\
Volatility Index (VIX) & 3,303 & 88,319 & Mar. 26, 2004 \\
DJIA Volatility Index (VXD) & 416 & 961 & Apr. 25, 2005 \\
S\&P 500 Three-Month Variance & 76 & 2,052 & May 18, 2004 \\
S\&P 500 Twelve-Month Variance & 0 & 60 & Mar. 23, 2006 \\
& & & \\
Panel B: The CBOE volatility options & & & \\
Nasdaq-100 volatility index (VXN) & 50 & 2,156 & Sep. 27, 2007 \\
Russell 2000 volatility index (RVX) & 220 & 11,543 & Sep. 27, 2007 \\
Volatility Index (VIX) & 87,349 & $2,240,946$ & Feb. 24, 2006 \\
& & & \\
Panel C: The Eurex volatility futures & & & \\
VDAX-NEWB Futures (FVDX) & 0 & 0 & Sep. 19, 2005 \\
VSMIB Futures (FVSM) & 0 & 0 & Sep. 19, 2005 \\
VSTOXXB) Futures (FVSX) & 30 & 0 & Sep. 19, 2005 \\
\hline
\end{tabular}

VIX, VXD, RVX, VXN, VDAX-NEW, VSMI and VSTOXX are all volatility indices based on different stock index options, which are designed to reflect investors' consensus view of future (30-day) expected stock market volatility by measuring the square root of the implied variance across corresponding options of a given time to expiration.

The CBOE Volatility Index, VIX, is calculated based on the prices of the S\&P 500 index (SPX) options.

The CBOE DJIA Volatility Index (VXD) is based on real-time prices of options on the 
Dow Jones Industrial Average (DJIA).

The CBOE Russell 2000 Volatility Index, more commonly referred to as RVX, is an upto-the-minute market estimate of expected volatility that is calculated by using real-time Russell 2000 Index (RUT) option bid/ask quotes.

The CBOE Nasdaq-100 Volatility Index (VXN) is calculated by using real-time Nasdaq100 Index (NDX) option bid/ask quotes.

VDAX-NEW is calculated based on the options of DAX that measures the performance of the Prime Standards 30 largest German companies in terms of order book volume and market capitalization.

VSMI is calculated based on options of the Swiss Market Index (SMI).

VSTOXX is calculated based on the Dow Jones EURO STOXX 50 real-time options prices, Dow Jones EURO STOXX 50 is Europe's leading Blue-chip index for the Eurozone. It provides a Blue-chip representation of supersector leaders in the Eurozone. The index covers 50 stocks from 12 Eurozone countries: Austria, Belgium, Finland, France, Germany, Greece, Ireland, Italy, Luxembourg, the Netherlands, Portugal and Spain. 


\section{Table 2}

\section{Summary Statistics for Three-month Variance Futures Contracts}

This table reports summary statistics of the three-month variance futures contracts traded in the CFE from May 18, 2004 to August 17, 2007.

\begin{tabular}{lrrrrrrr}
\hline Code & No. of & \multicolumn{2}{c}{ Period Covered } & \multicolumn{2}{c}{ Settle Price } & Open Interest & Volume \\
& Obs. & & & Mean & Std. & Mean & Mean \\
\hline U04 & 85 & $05 / 18 / 04$ & $09 / 17 / 04$ & 228.6 & 79.0 & 56 & 2.8 \\
Z04 & 149 & $05 / 18 / 04$ & $12 / 17 / 04$ & 282.7 & 107.9 & 97 & 3.3 \\
H05 & 211 & $05 / 18 / 04$ & $03 / 18 / 05$ & 278.2 & 119.2 & 145 & 2.5 \\
M05 & 274 & $05 / 18 / 04$ & $06 / 17 / 05$ & 283.4 & 103.4 & 143 & 2.4 \\
U05 & 252 & $09 / 20 / 04$ & $09 / 16 / 05$ & 220.0 & 75.2 & 264 & 10.2 \\
Z05 & 253 & $12 / 20 / 04$ & $12 / 16 / 05$ & 219.5 & 51.1 & 310 & 4.8 \\
H06 & 251 & $03 / 21 / 05$ & $03 / 17 / 06$ & 220.4 & 65.5 & 86 & 1.5 \\
M06 & 251 & $06 / 20 / 05$ & $06 / 16 / 06$ & 204.4 & 57.9 & 86 & 3.7 \\
U06 & 251 & $09 / 19 / 05$ & $09 / 15 / 06$ & 219.0 & 50.3 & 65 & 1.9 \\
Z06 & 251 & $12 / 19 / 05$ & $12 / 15 / 06$ & 195.2 & 64.1 & 95 & 2.3 \\
H07 & 250 & $03 / 20 / 06$ & $03 / 16 / 07$ & 195.6 & 64.2 & 345 & 7.3 \\
M07 & 249 & $06 / 20 / 06$ & $06 / 15 / 07$ & 200.9 & 50.9 & 322 & 5.6 \\
U07 & 227 & $09 / 22 / 06$ & $09 / 21 / 07$ & 227.2 & 65.3 & 418 & 7.4 \\
Z07 & 167 & $12 / 18 / 06$ & $12 / 21 / 07$ & 248.6 & 74.6 & 982 & 16.4 \\
H08 & 104 & $03 / 22 / 07$ & $03 / 21 / 08$ & 281.4 & 87.4 & 47 & 2.0 \\
M08 & 44 & $06 / 18 / 07$ & $06 / 20 / 08$ & 361.4 & 102.7 & 9 & 0.7 \\
\hline Average & & & & 241.6 & 76.2 & 217 & 4.7 \\
\hline
\end{tabular}




\section{Table 3}

\section{Summary Statistics of Fixed Time-to-maturity Three-month Variance Futures Prices}

This table reports summary statistics of levels and returns of the S\&P 500 index, VIX, VIX ${ }^{2}$, 30-day and three-month historical variance (HV), three fixed time-to-maturity three-month variance futures (VF) prices based on the market data between May 18, 2004 and August 17, 2007. The 30-day (three-month) historical variance is calculated based on the S\&P 500 index in the last 21 (63) trading days. The fixed time-to-maturity three-month variance futures prices are constructed by using the market data of available contracts with a linear interpolation technique. The three-, six- and nine-month-to-maturity variance futures are for 63, 126 and 189 trading days to maturity, respectively. The return (daily continuously compounded) is defined as the logarithm of the ratio between the price on the next day and the price on the current day.

Panel A: Summary statistics of levels

\begin{tabular}{l|ccc|cc|ccc} 
& S\&P 500 & VIX & VIX $^{2}$ & 30-d HV & 3-m HV & 3-m VF & $6-\mathrm{m} \mathrm{VF}$ & $9-\mathrm{m} \mathrm{VF}$ \\
\hline Mean & 1274.64 & 13.57 & 191.40 & 115.93 & 112.41 & 196.06 & 248.18 & 268.44 \\
Median & 1256.54 & 13.05 & 170.30 & 93.84 & 112.00 & 182.25 & 231.81 & 251.32 \\
Std Dev & 121.49 & 2.70 & 91.48 & 73.01 & 37.45 & 60.74 & 67.16 & 68.10 \\
Skew & 0.53 & 2.27 & 3.87 & 2.77 & 1.02 & 2.01 & 1.63 & 1.59 \\
Kurt & -0.66 & 9.02 & 22.84 & 12.03 & 3.00 & 6.74 & 2.66 & 2.44 \\
Min & 1063.23 & 9.89 & 97.81 & 32.83 & 49.16 & 109.00 & 161.32 & 180.84 \\
Max & 1553.08 & 30.83 & 950.49 & 638.10 & 310.35 & 546.52 & 548.65 & 570.57 \\
\hline
\end{tabular}

Panel B: Summary statistics of returns

\begin{tabular}{l|ccc|cc|ccc} 
& S\&P 500 & VIX & VIX $^{2}$ & $30-\mathrm{d}$ HV & $3-\mathrm{m} \mathrm{HV}$ & $3-\mathrm{m} \mathrm{VF}$ & $6-\mathrm{m} \mathrm{VF}$ & $9-\mathrm{m} \mathrm{VF}$ \\
\hline Mean & $0.03 \%$ & $0.05 \%$ & $0.11 \%$ & $0.16 \%$ & $0.08 \%$ & $0.06 \%$ & $0.03 \%$ & $0.03 \%$ \\
Median & $0.08 \%$ & $-0.47 \%$ & $-0.94 \%$ & $0.04 \%$ & $0.01 \%$ & $-0.51 \%$ & $-0.23 \%$ & $-0.07 \%$ \\
Std Dev & 0.007 & 0.060 & 0.120 & 0.118 & 0.045 & 0.060 & 0.038 & 0.029 \\
Skew & -0.362 & 0.986 & 0.986 & 2.749 & 3.074 & 0.760 & 0.473 & 0.461 \\
Kurt & 1.852 & 7.776 & 7.776 & 41.137 & 61.451 & 5.232 & 3.387 & 3.192 \\
Min & $-3.53 \%$ & $-29.99 \%$ & $-59.97 \%$ & $-55.33 \%$ & $-36.50 \%$ & $-37.27 \%$ & $-20.55 \%$ & $-14.44 \%$ \\
Max & $2.43 \%$ & $49.60 \%$ & $99.20 \%$ & $159.29 \%$ & $65.37 \%$ & $40.33 \%$ & $19.58 \%$ & $15.71 \%$ \\
\hline
\end{tabular}




\section{Table 4}

\section{Correlation Matrix of Fixed Time-to-maturity Three-month Variance Futures Prices}

This table reports the correlation matrix between levels and returns of the S\&P 500 index, VIX, VIX ${ }^{2}, 30$-day and three-month historical variance (HV), three fixed time-to-maturity three-month variance futures (VF) prices based on the market data between May 18, 2004 and August 17, 2007. The 30-day (three-month) historical variance is calculated based on the S\&P 500 index in the last 21 (63) trading days. The fixed time-to-maturity three-month variance futures prices are constructed by using the market data of available contracts with a linear interpolation technique. The three-, six- and nine-month-to-maturity variance futures are for 63, 126 and 189 trading days to maturity, respectively. The return (daily continuously compounded) is defined as the logarithm of the ratio between the price on the next day and the price on the current day.

Panel A: The correlation matrix between levels

\begin{tabular}{l|ccc|cc|ccc} 
& S\&P 500 & VIX & VIX $^{2}$ & 30-d HV & 3-m HV & 3-m VF & 6-m VF & 9-m VF \\
\hline S\&P 500 & 1.00 & & & & & & & \\
VIX & -0.09 & 1.00 & & & & & & \\
VIX & -0.02 & 0.98 & 1.00 & & & & & \\
30-d HV & 0.06 & 0.76 & 0.78 & 1.00 & & & & \\
3-m HV & -0.05 & 0.62 & 0.61 & 0.67 & 1.00 & & & \\
3-m VF & -0.25 & 0.92 & 0.90 & 0.71 & 0.67 & 1.00 & & \\
6-m VF & -0.41 & 0.77 & 0.74 & 0.49 & 0.54 & 0.93 & 1.00 & \\
9-m VF & -0.46 & 0.73 & 0.70 & 0.47 & 0.53 & 0.90 & 0.99 & 1.00 \\
\hline
\end{tabular}

Panel B: The correlation matrix between returns

\begin{tabular}{l|ccc|cc|ccc} 
& S\&P 500 & VIX & VIX $^{2}$ & 30-d HV & 3-m HV & 3-m VF & 6-m VF & 9-m VF \\
\hline S\&P 500 & 1.00 & & & & & & & \\
VIX & -0.81 & 1.00 & & & & & & \\
VIX & -0.81 & 1.00 & 1.00 & & & & & \\
30-d HV & -0.09 & 0.16 & 0.16 & 1.00 & & & & \\
3-m HV & -0.11 & 0.20 & 0.20 & 0.59 & 1.00 & & & \\
3-m VF & -0.81 & 0.87 & 0.87 & 0.18 & 0.23 & 1.00 & & \\
6-m VF & -0.81 & 0.81 & 0.81 & 0.09 & 0.15 & 0.90 & 1.00 & \\
9-m VF & -0.75 & 0.75 & 0.75 & 0.06 & 0.13 & 0.82 & 0.92 & 1.00 \\
\hline
\end{tabular}


Table 5

\section{The Relation Between Variance Futures Prices and VIX ${ }^{2}$}

We preform regressions of three-, six- and nine-month-to-maturity variance futures prices against VIX ${ }^{2}$ and three-month (30-day) historical variance (HV). This table reports the intercepts and coefficients. The numbers in parentheses are t-statistics. The results in the table indicate that all linear regressions are significant at the one percent critical level.

\begin{tabular}{cccc} 
Variable & Intercept & Coefficient & $R^{2}$ \\
\hline \multirow{4}{*}{ Panel A: Three-month-to-maturity } & Variance Futures Prices & \\
& & & \\
VIX & & & \\
& $(38.35)$ & $(59.95)$ & \\
3-month HV & 74.11 & 1.0848 & $44.5 \%$ \\
& $(14.83)$ & $(25.71)$ & \\
30-day HV & 127.40 & 0.5923 & $50.7 \%$ \\
& $(45.50)$ & $(28.97)$ & \\
\hline
\end{tabular}

Panel B: Six-month-to-maturity Variance Futures Prices

$\begin{array}{lccc}\text { VIX }^{2} & 143.86 & 0.5450 & 55.1 \% \\ 3 \text { 3-month HV } & (39.41) & (31.67) & \\ & 138.89 & 0.9722 & 29.4 \% \\ \text { 30-day HV } & (22.23) & (18.44) & \\ & 196.24 & 0.4480 & 23.7 \% \\ & (50.96) & (15.94) & \end{array}$

Panel C: Nine-month-to-maturity Variance Futures Prices

$\begin{array}{lccc}\text { VIX }^{2} & 168.12 & 0.5241 & 49.6 \% \\ 3 \text { 3-month HV } & (42.85) & (28.33) & \\ & 159.76 & 0.9668 & 28.3 \% \\ \text { 30-day HV } & (25.02) & (17.94) & \\ & 218.15 & 0.4338 & 21.6 \% \\ & (55.13) & (15.01) & \end{array}$




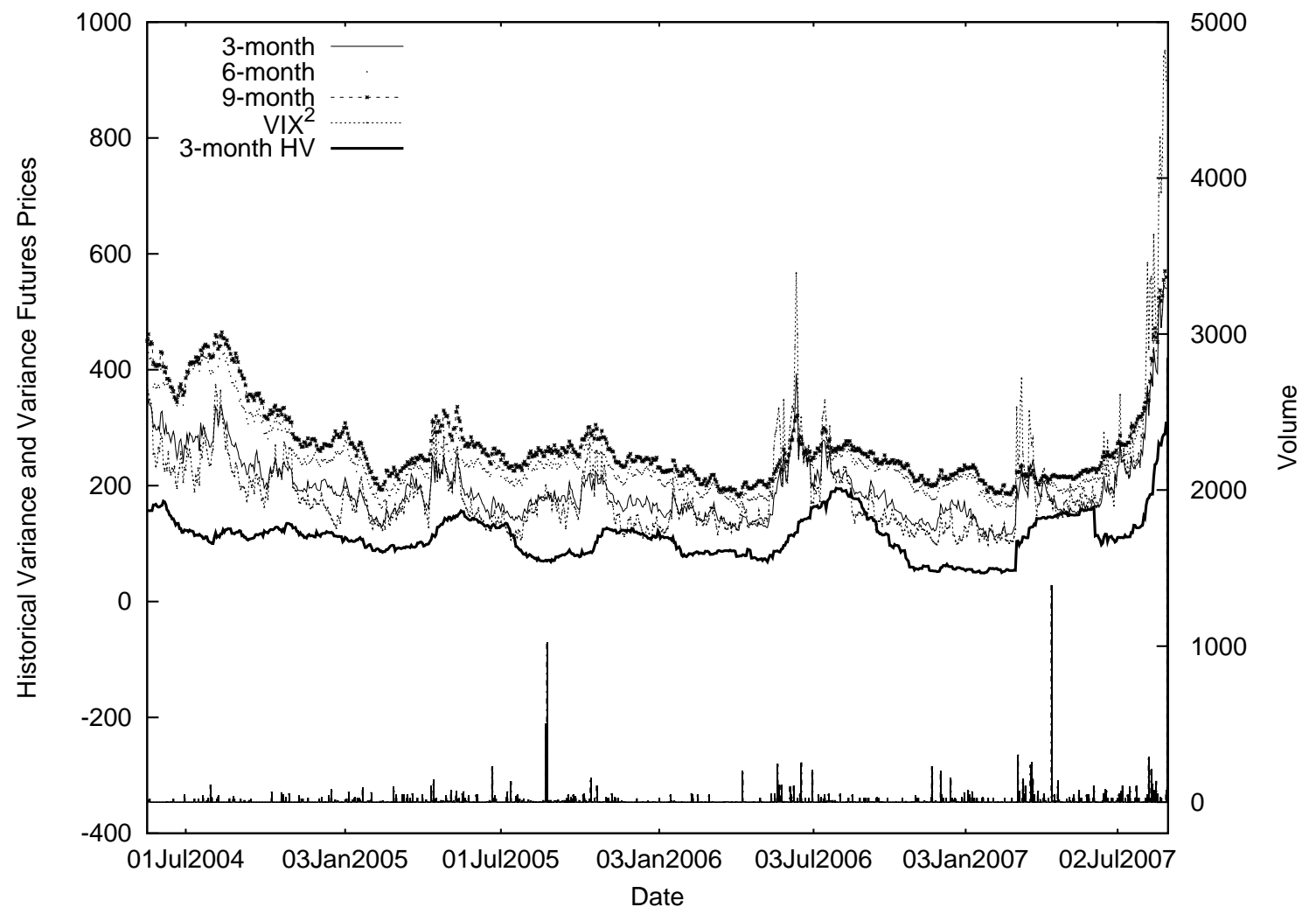

Figure 1: The three-month historical variance (HV), VIX ${ }^{2}$ and variance futures prices with three fixed time-to-maturities between May 18, 2004 and August 17, 2007. The threemonth HV is computed from the closing level of the S\&P 500 index in the last 63 trading days. The fixed time-to-maturity variance futures are constructed by using the market data of available contracts with a linear interpolation technique. The vertical bar chart shows the trading volume of futures of all maturities on each day. 

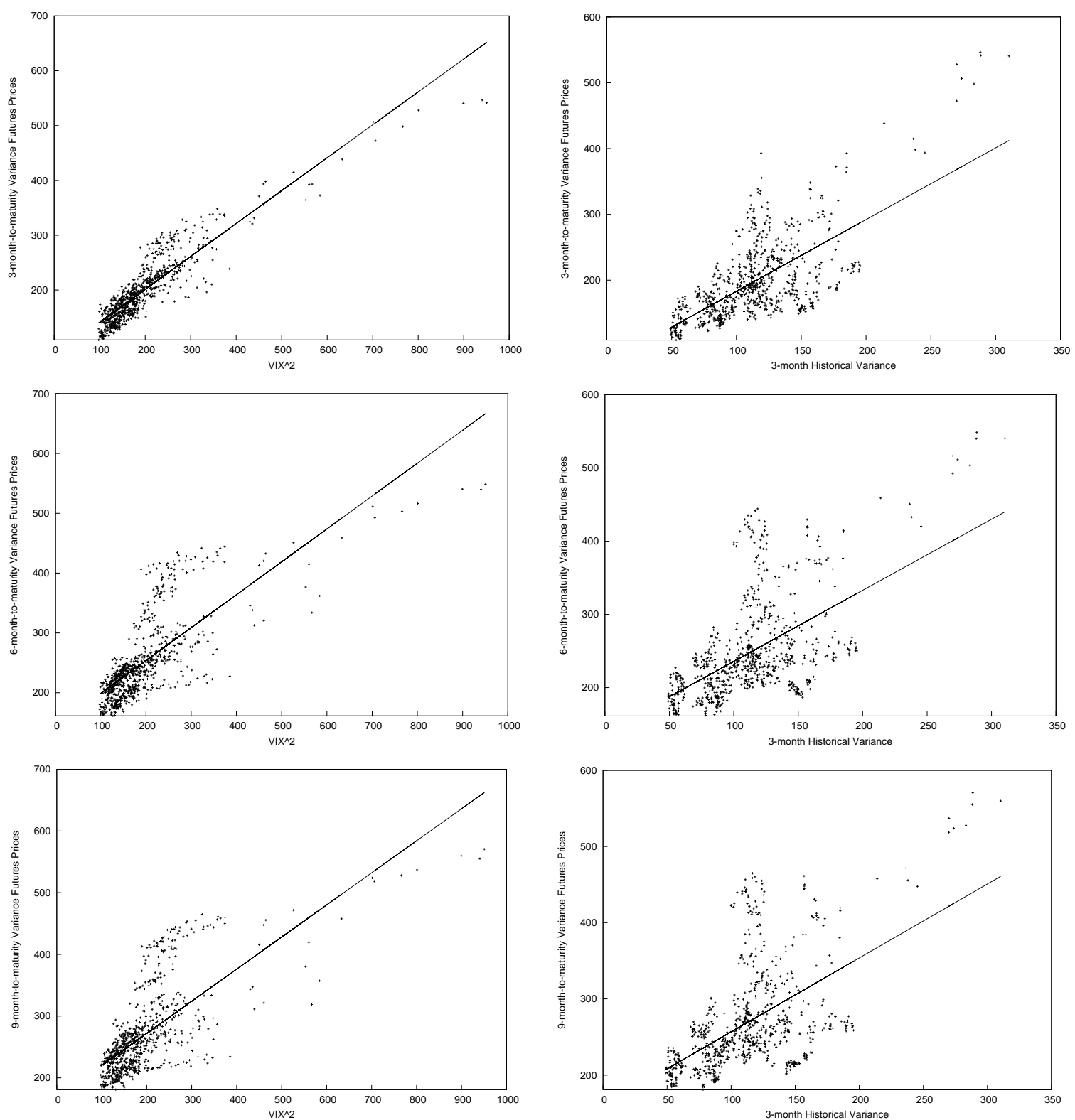

Figure 2: The relation between fixed time-to-maturity variance futures prices and VIX ${ }^{2}$ or three-month historical variance. The dots are market prices between May 18, 2004 and August 17, 2007. The solid lines are the results from linear regressions in Table 5. 

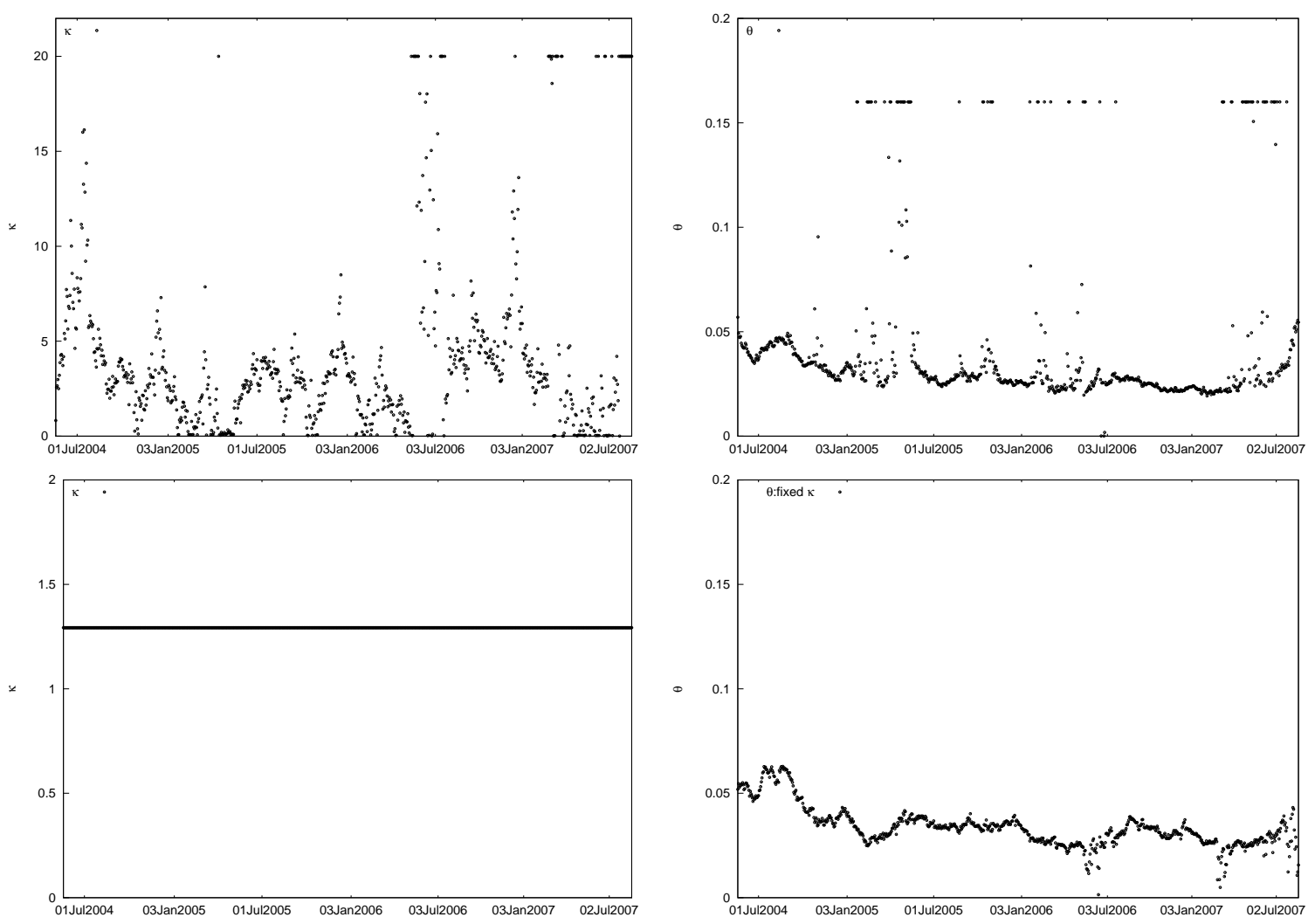

Figure 3: The values of variance mean-reversion parameters: mean reverting speed, $\kappa$, and long-term mean level, $\theta$, between May 18, 2004 and August 17, 2007. The parameters are calibrated daily by using the market prices of four variance futures with different maturity dates. The top two graphs are the calibrated results with a floating $\kappa$ and $\theta$. The lower two graphs are the fixed $\kappa=1.2929$ and the calibrated floating $\theta$. 

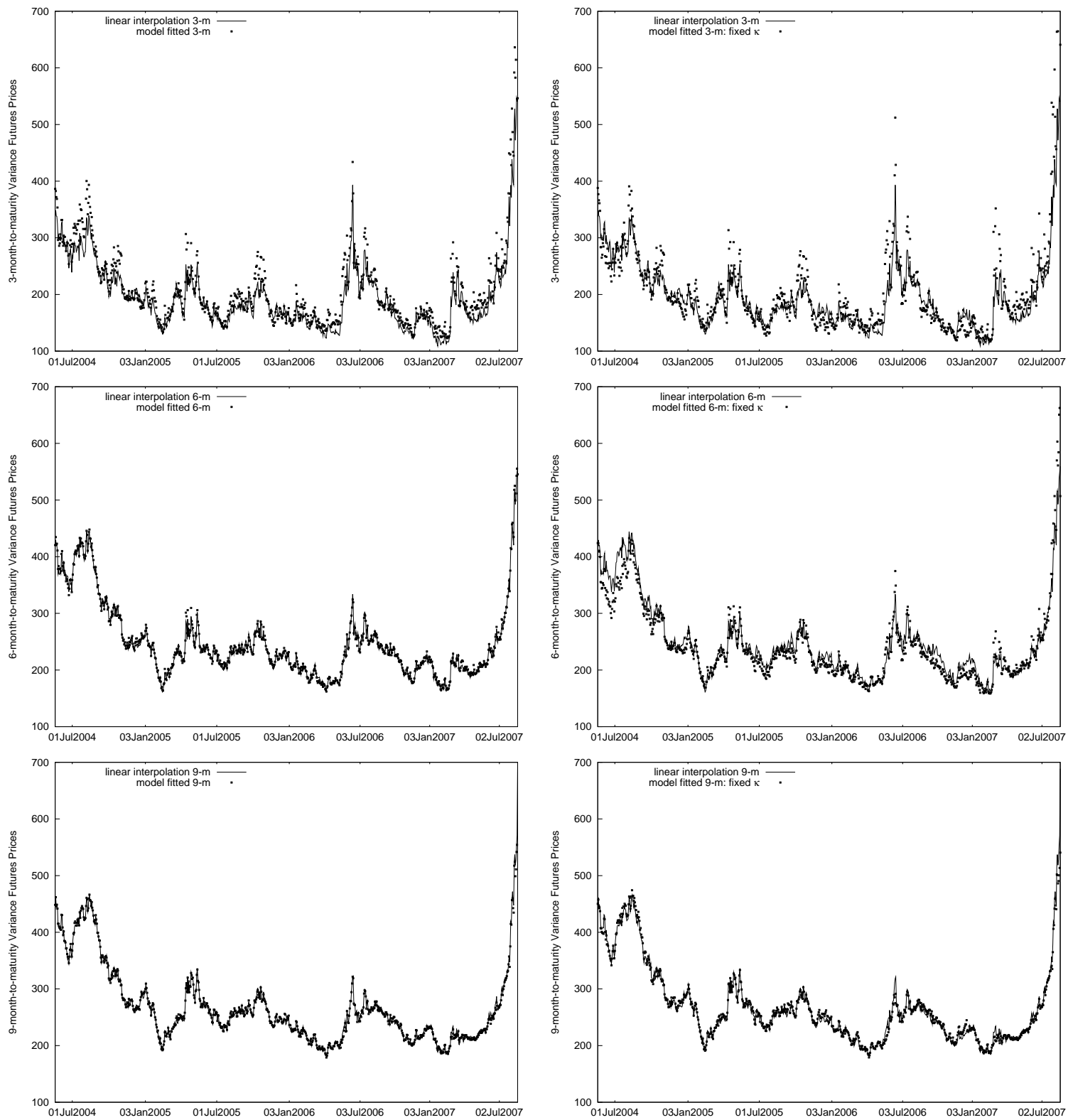

Figure 4: The prices of three-, six- and nine-month-to-maturity variance futures constructed with two different methods between May 18, 2004 and August 17, 2007. One is to use the linear interpolation technique with market prices of available variance futures. The other one is to compute by using variance futures pricing model with parameters $(\kappa, \theta)$ calibrated on the same day. The left three figures are for floating $\kappa$ and $\theta$. The right three figures are for fixed $\kappa=1.2929$ and floating $\theta$. 

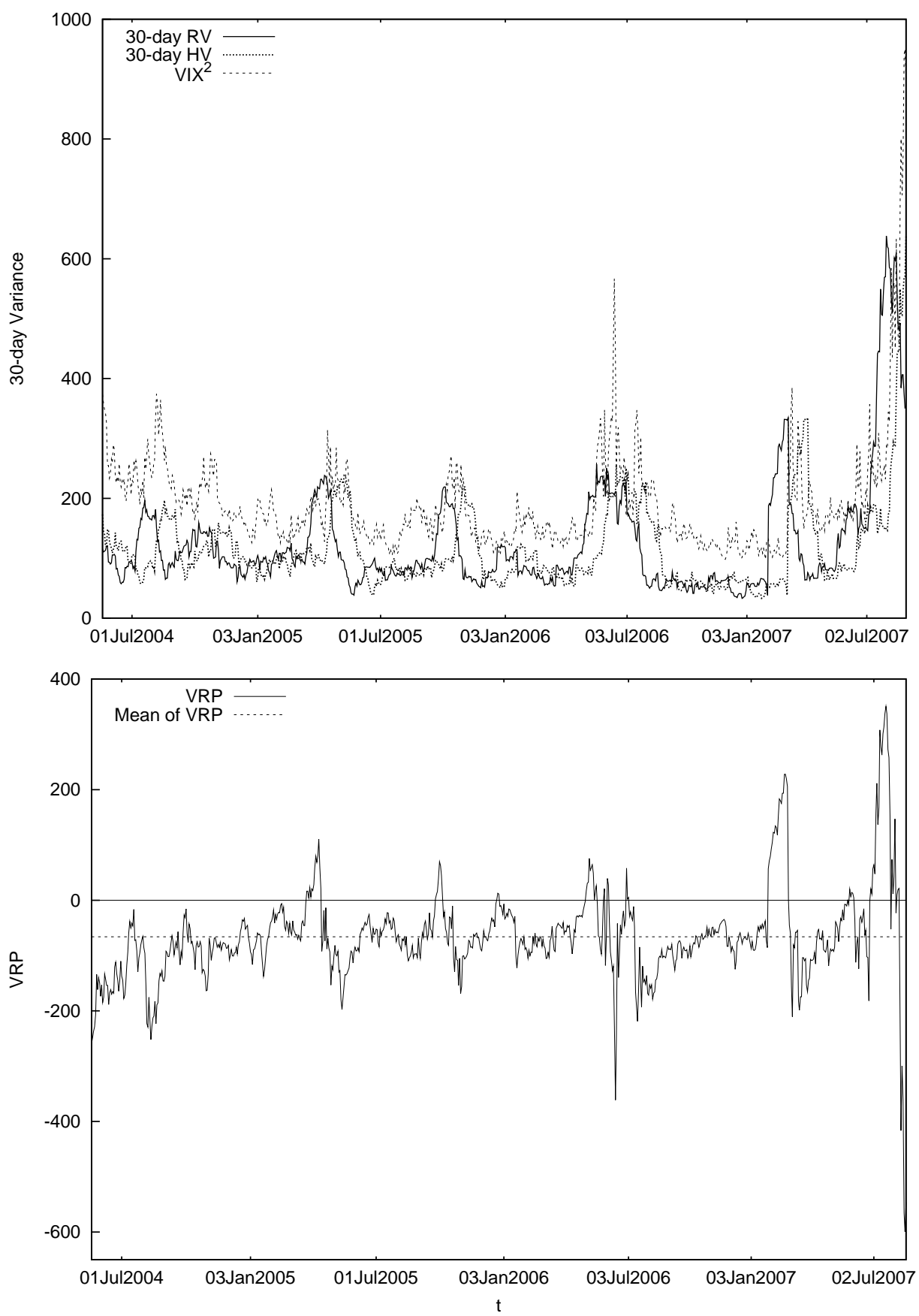

Figure 5: The variance risk premium embedded in the SPX options market between May 18, 2004 and August 17, 2007. The top graph shows the 30-day historical variance, $H V_{t}$, realized variance, $R V_{t}$, and $V I X_{t}^{2}$. The lower graph shows the variance risk premium (VRP), measured by the difference between the 30-day realized variance and $V I X^{2}$, i.e., $V R P_{t}=R V_{t}-V I X_{t}^{2}$. The average value of the variance risk premium is -66.0413 . 

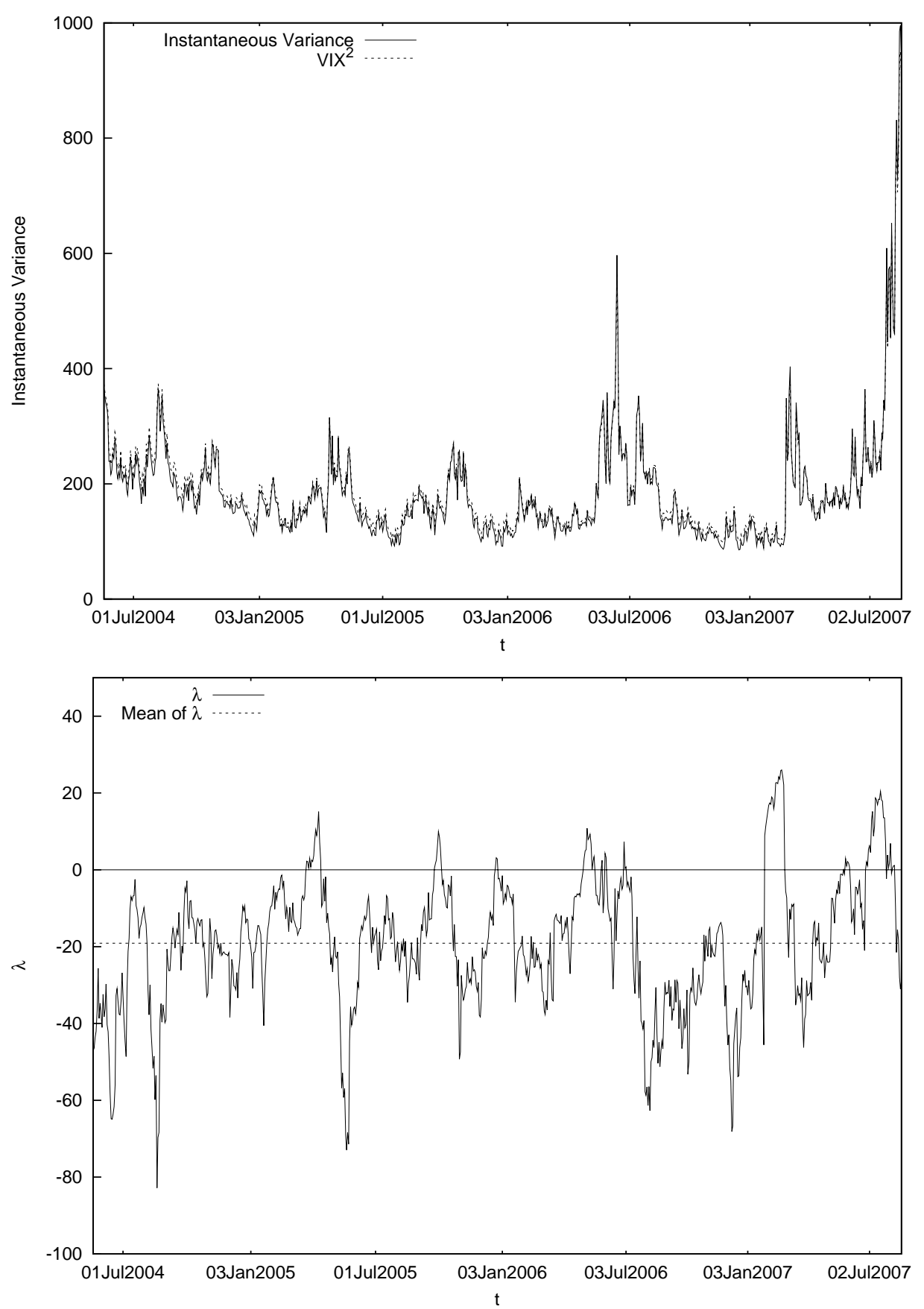

Figure 6: The market price of volatility risk in the SPX model with Heston (1993) between May 18, 2004 and August 17, 2007. The top graph shows $V I X_{t}^{2}$ and the instantaneous variance, $V_{t}$, calculated from the observable $V I X_{t}$. The lower graph shows the market price of volatility risk, $\lambda$, calculated from $V R P_{t}, V_{t}$ and calibrated floating $\theta_{t}$ with fixed $\kappa=1.2929$. The average value of $\lambda$ is -19.1184 . 